\title{
Ingestion of Dried-bonito Broth Ameliorates Blood Fluidity in Humans
}

\author{
Yoshizu Nozawa, ${ }^{, a}$ Taichi Ishizaki, ${ }^{a}$ Motonaka Kuroda, ${ }^{a}$ Kikue Takahashi, ${ }^{b}$ \\ Shukuko Ebihara, ${ }^{b}$ and Teiji Itoh ${ }^{c}$
}

${ }^{a}$ Processed Food Development \& Technology Center, Ajinomoto Co., Inc., 1-1 Suzuki-cho, Kawasaki, 210-8681, Japan, ${ }^{b}$ Chiyoda Paramedical Care Clinic, 3-5-5 Uchikanda, Tokyo, 101-0047, Japan, and ${ }^{c}$ CPCC Co., Ltd., 3-5-5 Uchikanda, Tokyo, 101-0047, Japan

(Received May 11, 2007; Accepted August 9, 2007; Published online August 10, 2007)

To elucidate a physiological function of dried-bonito broth (DBB) on blood fluidity and oxidative stress, we performed a randomized double-blind placebo-controlled study in twenty-four healthy adult subjects. The subjects ingested DBB or a placebo for four weeks, and blood fluidity and oxidative stress were measured before and after ingestion. Blood fluidity was measured using a microchannel array flow analyzer by the passage time of $100 \mu l$ of heparinized whole blood through the microchannel array, while oxidative stress was evaluated as a level of derivative of reactive oxygen metabolites (d-ROMs) by a free radical analysis system (FRAS). DBB ingestion significantly shortened the blood passage time from $55.4 \pm 3.4$ to $47.6 \pm 2.0 \mathrm{sec}$ (mean $\pm \mathrm{SEM}, p<0.05$ ), while no significant change was observed in the placebo group ( $52.4 \pm 3.4$ to $51.4 \pm 2.6 \mathrm{sec}$, mean \pm SEM) indicating that DBB ameliorated blood fluidity. The level of d-ROMs, known as a biomarker of oxidative stress, significantly decreased after DBB ingestion from $337.2 \pm 18.5$ to $316.5 \pm 12.9$ Carrotelli units (Carr. U.) (mean \pm SEM, $p<0.05$ ), suggesting that DBB reduced oxidative stress. Among subjects with a d-ROMs score $>320$, regarded as being in a state of oxidative stress, changes in blood fluidity tended to correlate with changes in d-ROMs score $(\rho=0.55, p=0.06)$, showing that blood fluidity may have improved in subjects whose oxidative stress was markedly decreased. These results also showed a possibility that DBB ingestion improved blood fluidity by decreasing oxidative stress. In previous studies, daily DBB ingestion improved various fatigue-related symptoms, so we investigated the effect of DBB on fatigue-related symptoms via a questionnaire survey in the present study. The result of this survey showed that symptoms of shoulder stiffness and visual fatigue were improved only in the DBB group $(p<0.05, p<0.1$, respectively). Insufficient blood circulation is considered to lead to the development of shoulder stiffness, visual fatigue, and other fatigue-related symptoms. Based on these findings, we considered that dietary intake of DBB may improve blood fluidity by reducing oxidative stress and thus might protect against fatigue.

Key words — dried-bonito broth, blood fluidity, oxidative stress, visual fatigue, shoulder stiffness, human

\section{INTRODUCTION}

Capillary vessels are responsible for constant oxygen and carbon dioxide gas exchange and nutrient transport, enabling the life-support of all tissues. ${ }^{1)}$ Insufficient blood circulation has been found to lead to the development of various pathogeneses such as shoulder stiffness, neck pain, poor circulation, and dark skin circles. ${ }^{2-4)}$ Furthurmore, impaired microvascular perfusion could contribute to

*To whom correspondence should be addressed: Processed Food Development \& Technology Center, Ajinomoto Co., Inc., 1-1 Suzuki-cho, Kawasaki, 210-8681, Japan. Tel.: +81-44-223-4122; Fax: +81-44-246-6241; E-mail: yoshizu_nozawa@ajinomoto.com the development and progression of diseases such as high blood pressure, arteriosclerosis, and thrombus syndrome. Thus, the improvement of blood fluidity is important for preventing these pathogeneses and may be important in decreasing fatigue-related subjective symptoms.

Beef and chicken bouillons have been commonly used worldwide as a flavoring base in many cuisines. In Japan, dried-bonito broth (DBB), which has a specific taste and flavor, has been more frequently used than beef and chicken bouillons for soup stock. Dried bonito and DBB have been traditionally considered nutritional supplements that promote recovery from fatigue. Studies have confirmed that DBB shows various physiological functions in both animals and humans. Animal stud- 
ies showed that DBB administration aided recovery from physical fatigue, ${ }^{5)}$ while in human studies, the daily ingestion of DBB improved mood states ${ }^{6}$ ) and subjective symptoms of visual fatigue. ${ }^{7-9)}$ In addition, DBB was shown to have an improving effect on the skin of subjects whose skin tends to be dry and rough by maintaining moisture levels in the skin. ${ }^{10)}$ However, even though DBB has been found to have these physiological effects, an understanding of why its effectiveness is so wide-ranging has remained elusive.

To investigate whether daily DBB ingestion improved blood fluidity, we used a microchannel array flow analyzer (MC-FAN), which enabled us to take into account erythrocytes deformability, platelet aggregation, and leukocyte adhesiveness. The time required for a specified volume of blood to pass through microchannels that simulate human blood vessels is measured to evaluate blood fluidity by this system, ${ }^{11-13)}$ which is widely used for the evaluation of the physiological function of foods and supplements ${ }^{14-17)}$ and in disease models. ${ }^{18,19)}$ The fatigue-related subjective symptoms were also evaluated by a questionnaire survey during the test diet ingestion period.

Erythrocytes form the largest group of cells in the blood, and they are highly deformable. The membrane plays an important role in maintaining the flexibility for normal erythrocyte deformability, which was reported to be damaged by reactive oxygen. ${ }^{20,21)}$ Oxidized erythrocytes would be more prone to form aggregates and increase the viscosity of blood flow in the microcirculation. ${ }^{16,22)}$ Since the antioxidative effect is considered to be a factor in improving blood fluidity, we investigated deriva- tives of reactive oxygen metabolites (d-ROMs) using a free radical analysis system. ${ }^{23-25)}$

In the present study, we measured blood fluidity, the level of oxidative stress marker before and after the ingestion period, and the change of subjective symptoms assessed by a daily questionnaire each night. Based on the results obtained, we discuss why DBB is effective to multiple symptoms.

\section{MATERIALS AND METHODS}

Study Design and Protocol — This study was conducted in accordance with the Declaration of Helsinki, and was approved by the ethics committee of the institutional review board of CPCC Co., Ltd. (Tokyo, Japan) Written informed consent was obtained from each subject participating in the study. A randomized doubleblind placebo-controlled study was performed in twenty-four healthy adult subjects aged $42.0 \pm 2.0$ (mean $\pm \mathrm{SEM}$ ) years. Each group was comprised of seven male and five female volunteers. Subjects were screened before the start of this study and randomly divided into two groups so that there were no differences in blood fluidity measured by MC-FAN. The subjects ingested the DBB or placebo for four weeks, and blood fluidity and oxidative stress were measured before and after the ingestion period. The subjects filled in a questionnaire during the study period. The study protocol is outlined in Fig. 1.

Test Diet — The compositions of the test diets, DBB, and the placebo are shown in Table 1. Commercial dried-bonito broth, named "Hondzukuri ichiban-dashi” (Ajinomoto Co., Inc., Tokyo Japan),

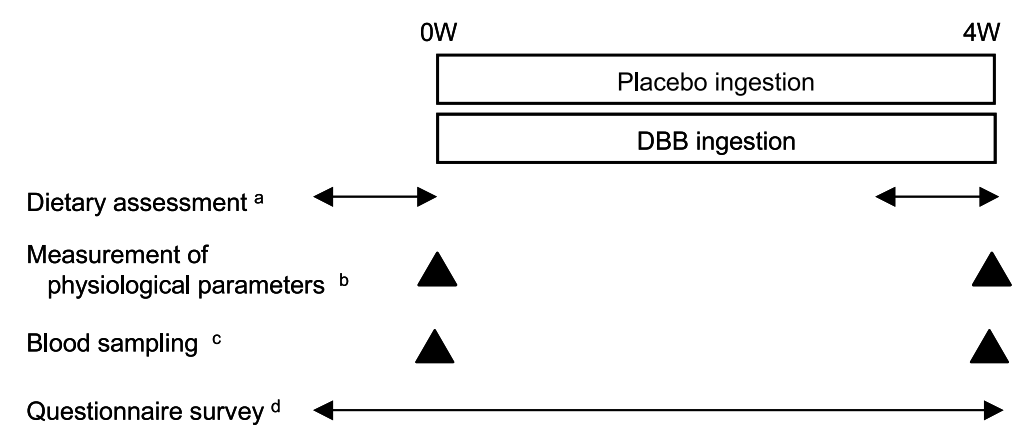

Fig. 1. Study Protocol

a; All meals were recorded beginning three days before blood testing. The subjects also filled in a questionnaire that contained questions regarding their daily appetite, food and water intake, urination, and defecation. b; Body weight, percentage body fat, and blood pressure were measured before and after test diet ingestion. c; Blood testing was performed before and after the ingestion period to evaluate the blood fluidity and oxidative stress marker. d; Each night, the subjects filled in a questionnaire that asked about subjective symptoms (frequency of feeling fatigue, anxiety and stress, visual fatigue, shoulder stiffness, skin condition), beginning three days before initiation of the study and continuing until completion of the study. 
Table 1. Nutrient Compositions of the Test Diets (Per $100 \mathrm{ml}$ )

\begin{tabular}{llcc}
\hline \hline & & Placebo $^{a)}$ & DBB $^{b)}$ \\
\hline Energy & $(\mathrm{kcal})$ & 4.2 & 16 \\
Protein & $(\mathrm{g})$ & - & 3.6 \\
Lipid & $(\mathrm{g})$ & - & 0.1 \\
Carbohydrate & $(\mathrm{g})$ & 0.3 & 0.1 \\
Ash content & $(\mathrm{g})$ & 0.3 & 0.9 \\
Water content & $(\mathrm{g})$ & 99.6 & 95.3 \\
Sodium & $(\mathrm{mg})$ & 120 & 123 \\
\hline
\end{tabular}

a) Placebo: Dried-bonito flavor, caramel, and sodium chloride were dissolved in water. b) DBB: Dried-bonito broth; - : Not calculated because the value was lower than $0.1 \mathrm{~g} / 100 \mathrm{~g}$.

Table 2. Nutrient Composition of DBB

\begin{tabular}{llc}
\hline \hline Nutrient & & Amount \\
\hline Protein (include peptides) & $(\mathrm{mg} / \mathrm{ml})$ & 20.6 \\
Free amino acid & $(\mathrm{mg} / \mathrm{ml})$ & 10.4 \\
$\quad$ taurine & $(\mathrm{mg} / \mathrm{ml})$ & 1.2 \\
histidine & $(\mathrm{mg} / \mathrm{ml})$ & 6.4 \\
anserine & $(\mathrm{mg} / \mathrm{ml})$ & 0.9 \\
Organic acid & & \\
$\quad$ lactic acid & $(\mathrm{mg} / \mathrm{ml})$ & 8.2 \\
Minerals & & \\
iron & $(\mu \mathrm{g} / \mathrm{ml})$ & 2.0 \\
magnesium & $(\mu \mathrm{g} / \mathrm{ml})$ & 243.3 \\
sodium & $(\mathrm{mg} / \mathrm{ml})$ & 2.3 \\
potasium & $(\mathrm{mg} / \mathrm{ml})$ & 1.4 \\
calcium & $(\mu \mathrm{g} / \mathrm{ml})$ & 36.7 \\
Creatine & $(\mathrm{mg} / \mathrm{ml})$ & 0.9 \\
Creatinine & $(\mathrm{mg} / \mathrm{ml})$ & 1.2 \\
\hline
\end{tabular}

produced via a hot-water extraction process from dried bonito, was used as an active dietary supplement. The nutrient composition of DBB is shown in Table 2. The placebo consisted of dried-bonito flavor, caramel, and sodium chloride, and was prepared so that the two test diets were indistinguishable. The subjects ingested $125 \mathrm{ml}$ of the diet every morning in addition to their regular diet for four weeks.

Dietary Assessment — All meals were recorded for three days before blood testing (Fig. 1). According to questionnaire, intake of energy, carbohydrate, protein and fat were calculated from the diet records using calculation software (Excel Eiyo-kun, Kenpakusha, Tokyo, Japan). Furthermore, the intake of rice, cereal, fish, meat, egg, pulse, milk, potato, vegetable and fruits were evaluated. The subjects also filled in a questionnaire that asked about their daily appetite, food and water intake, urination, and defecation.
Measurement of Physiological Parameters

Body weight, percentage body fat (InBody 3.2, Biospace Inc., Seoul, Korea), and blood pressure (Digital blood measurement TM-2655P, A\&D Co., Ltd., Tokyo, Japan) were measured before and after test diet ingestion (Fig. 1).

Measurement of Blood Fluidity — After blood testing, we measured blood fluidity with a MCFAN using the method described by Kikuchi et al. (Fig. 1). ${ }^{11,12,26)}$ Blood samples were collected in heparinized tubes for anticoagulation in the morning. Blood was then forced to flow through microchannels (Bloody 7-7, 7854 flow channels, $7 \mu \mathrm{m}$ width chip) and measured by an MC-FAN KH-7 model (Hitachi Haramachi Electronics Co., Ltd., Ibaraki, Japan). The passage time of each blood sample was measured twice, and the mean value was regarded as the blood passage time, which was an index of blood fluidity.

Measurement of Oxidative Stress Marker

Blood testing was performed, ultracentrifuged and evaluated photometrically by using a free radical analysis system (FRAS, Wismerll Co., Ltd., Italy; Fig. 1). The d-ROMs were measured to monitor oxidative stress in serum. ${ }^{23-25,27)}$ The d-ROMs score reflects the level of hydroperoxides of lipids, proteins, amino acids, and nucleic acids oxidized by free radicals, and is known as an oxidative injury index. The results of the d-ROMs test were expressed in arbitrary units called Carratelli units (Carr. U.), where 1 Carr. U. corresponds to $0.08 \mathrm{mg} / 100 \mathrm{ml}$ $\mathrm{H}_{2} \mathrm{O}_{2}$. ${ }^{28)}$

Questionnaire on Subjective Symptoms Each night, the subjects filled in a questionnaire on subjective symptoms, beginning three days before initiation of the study and continuing until completion of the study (Fig. 1). In the questionnaire, items related to fatigue such as frequency of feeling fatigue, anxiety and stress, visual fatigue, shoulder stiffness and skin condition were included.

Statistical Analysis — All values were presented as the mean \pm standard error of the mean (mean \pm SEM). Dietary assessment, physiological parameters, blood fluidity and oxidative stress were analyzed using a paired $t$ test to compare data before and after the 4-week period of ingestion. The relation between the change of blood fluidity and that of oxidative stress marker was analyzed by Spearman's correlation coefficient test. Regarding the questionnaire results, replies related to subjective symptoms were summed per week in each test diet group, and changes between week 1 and other 
weeks were compared. Frequencies were analyzed by the Wilcoxon rank sum test. All data analysis were conducted with the StatView software package (StatView 5 for SAS Institute, Inc., Cary, NC, U.S.A.). $p<0.05$ was regarded as significant.

\section{RESULTS}

All twenty-four subjects completed the study and were included in the analysis.

\section{Results of Dietary Assessment}

The results of measuring the the intake of energy, carbohydrate, protein and fat are shown in Table 3. The amounts of daily intake before blood testing showed no significant differences between before and 4-week after ingestion period (Table 3). We have also analyzed the amounts of daily intake of rice, cereals, fish, meat, egg, pulse, milk, potato, vegetable, and fruits which can be calculated by the soft used, and found that there were no significant differences between before and after ingestion period (data not shown). As a result of questionnaire analysis by Wilcoxon rank sum test, we found that there were no significant changes in the frequency of the daily appetite, food and water intake (data not shown). These results indicated that the subjects' dietary habit has not changed during the study.

\section{Results of Physiological Parameters}

There were no significant changes in the physiological parameters of body weight, percentage body fat, and blood pressure before and after the ingestion of placebo or DBB (Table 4).

Table 3. Results of Daily Intake of Energy, Carbohydrate, Protein, and Fat Before Blood Testing (Per Day)

\begin{tabular}{|c|c|c|}
\hline & Placebo & DBB \\
\hline \multicolumn{3}{|c|}{ Energy intake (kcal) } \\
\hline before & $2047.4 \pm 167.9$ & $1932.8 \pm 126.8$ \\
\hline after & $1938.9 \pm 114.3$ & $1893.1 \pm 117.6$ \\
\hline \multicolumn{3}{|c|}{ Carbohydrate (g) } \\
\hline before & $259.3 \pm 25.1$ & $235.1 \pm 17.3$ \\
\hline after & $255.1 \pm 19.0$ & $256.9 \pm 21.0$ \\
\hline \multicolumn{3}{|l|}{ Protein (g) } \\
\hline before & $77.5 \pm$ & $78.2 \pm$ \\
\hline after & $70.1 \pm$ & $70.8 \pm$ \\
\hline \multicolumn{3}{|l|}{ Fat $(g)$} \\
\hline before & $68.7 \pm$ & $67.7 \pm$ \\
\hline after & $65.9 \pm$ & $59.7 \pm$ \\
\hline
\end{tabular}

Values are mean $\pm \operatorname{SEM}(n=12)$.

\section{Effects on Blood Fluidity}

The passage time of $100 \mu \mathrm{l}$ of whole blood and the time points when 25,50 , and $75 \mu 1$ of the blood sample passed were measured before and after the ingestion period in the placebo and DBB groups. Measurement for one subject in the placebo group failed, so analysis was performed on the data from the eleven remaining subjects. In the placebo group, no significant changes were observed in any passage time during the ingestion period (Table 5). In contrast, the $50-\mu 1$ passage time tended to be shorter after DBB ingestion compared to that before ingestion $(p<0.1$; Table 5), and the $75-\mu 1$ and $100-\mu$ passage times were significantly shortened in the DBB group $(p<0.05$; Table 5).

Table 4. Results of Physiological Parameters Before and After Ingestion

\begin{tabular}{lcc}
\hline \hline & Placebo & DBB \\
\hline $\begin{array}{l}\text { Body weight (kg) } \\
\text { before }\end{array}$ & $65.3 \pm 4.2$ & $66.5 \pm 4.3$ \\
$\quad$ after & $65.5 \pm 4.2$ & $66.8 \pm 4.4$ \\
$\begin{array}{l}\text { Percentage body fat }(\%) \\
\text { before }\end{array}$ & \\
$\quad$ after & $22.4 \pm 2.9$ & $25.3 \pm 1.6$ \\
$\begin{array}{l}\text { Systolic blood pressure (mmHg) } \\
\text { before }\end{array}$ & $118.5 \pm 6.2$ & $113.3 \pm 5.2$ \\
$\quad$ after & $121.4 \pm 6.0$ & $114.8 \pm 5.2$ \\
Diastolic blood pressure (mmHg) & & \\
$\quad$ before & $74.7 \pm 4.2$ & $72.6 \pm 4.5$ \\
$\quad$ after & $78.6 \pm 3.7$ & $75.2 \pm 4.2$ \\
\hline
\end{tabular}

Values are mean $\pm \operatorname{SEM}(n=12)$.

Table 5. Results of Blood Passage Time and d-ROMs Score before and after Ingestion

\begin{tabular}{|c|c|c|c|}
\hline & & Placebo & DBB \\
\hline \multicolumn{4}{|c|}{ Blood passage time (sec) } \\
\hline \multirow{2}{*}{$25 \mu 1$} & before & $10.1 \pm 0.5$ & $10.3 \pm 0.4$ \\
\hline & after & $10.5 \pm 0.4$ & $9.9 \pm 0.3$ \\
\hline \multirow{2}{*}{$50 \mu 1$} & before & $22.1 \pm 1.4$ & $22.4 \pm 1.0$ \\
\hline & after & $22.4 \pm 0.9$ & $20.9 \pm 0.6]^{7}$ \\
\hline \multirow{2}{*}{$75 \mu 1$} & before & $37.7 \pm 3.9$ & $37.0 \pm 1.97$ \\
\hline & after & $35.7 \pm 1.5$ & $33.3 \pm 1.1$ \\
\hline \multirow{2}{*}{$100 \mu \mathrm{l}$} & before & $52.4 \pm 3.4$ & $55.4 \pm 3.4 \neg$ \\
\hline & after & $51.4 \pm 2.6$ & $47.6 \pm 2.0]^{*}$ \\
\hline \multicolumn{4}{|c|}{ d-ROMs score (Carr. U.) } \\
\hline & before & $321.5 \pm 17.1$ & $337.2 \pm 18.5 \neg$ \\
\hline . & after & $306.6 \pm 13.5$ & $316.5 \pm 12.9\rfloor^{*}$ \\
\hline
\end{tabular}

The passage times of $25,50,75$, and $100 \mu 1$ of whole blood, and derivatives of reactive oxygen metabolites (d-ROMs) are shown. Values are mean $\pm \operatorname{SEM}(n=11-12) .{ }^{*} p<0.05 v s$. before ingestion. ${ }^{\#} p<0.1 v s$. before ingestion. 
Table 6. Changes in Subjective Symptoms after Placebo or DBB Ingestion (Number of Replies Related to Frequency of Feeling Fatigue)

\begin{tabular}{|c|c|c|c|c|c|c|c|c|}
\hline & \multicolumn{4}{|c|}{ Placebo } & \multicolumn{4}{|c|}{ DBB } \\
\hline & 1 week & 2 weeks & 3 weeks & 4 weeks & 1 week & 2 weeks & 3 weeks & 4 weeks \\
\hline Very frequently & 2 & 0 & 5 & 0 & 1 & 0 & 3 & 0 \\
\hline Frequently & 24 & 24 & 28 & 18 & 24 & 14 & 16 & 25 \\
\hline Moderate & 40 & 47 & 38 & 43 & 50 & 55 & 43 & 48 \\
\hline Rarely & 17 & 12 & 12 & 19 & 9 & 14 & 22 & 11 \\
\hline Not at all & 1 & 1 & 1 & 4 & 0 & 1 & 0 & 0 \\
\hline
\end{tabular}

The frequency of "felt fatigue" was not changed significantly in week 2, 3, and 4 compared to week 1.

Table 7. Changes in Subjective Symptoms after Placebo or DBB Ingestion (Number of Replies Related to Anxiety and Stress)

\begin{tabular}{|c|c|c|c|c|c|c|c|c|}
\hline & \multicolumn{4}{|c|}{ Placebo } & \multicolumn{4}{|c|}{ DBB } \\
\hline & 1 week & 2 weeks & 3 weeks & 4 weeks & 1 week & 2 weeks & 3 weeks & 4 weeks \\
\hline Very frequently & 0 & 0 & 2 & 0 & 0 & 1 & 2 & 0 \\
\hline Frequently & 3 & 6 & 10 & 7 & 10 & 5 & 18 & 7 \\
\hline Moderate & 56 & 60 & 52 & 52 & 61 & 61 & 53 & 55 \\
\hline Rarely & 23 & 14 & 17 & 18 & 13 & 17 & 10 & 22 \\
\hline Not at all & 2 & 4 & 3 & 7 & 0 & 0 & 1 & 0 \\
\hline
\end{tabular}

The frequency of "felt anxiety and stress" was not changed significantly in week 2, 3, and 4 compared to week 1 .

Table 8. Changes in Subjective Symptoms after Placebo or DBB Ingestion (Number of Replies Related to Skin Condition)

\begin{tabular}{|c|c|c|c|c|c|c|c|c|}
\hline & \multicolumn{4}{|c|}{ Placebo } & \multicolumn{4}{|c|}{ DBB } \\
\hline & 1 week & 2 weeks & 3 weeks & $\overline{4 \text { weeks }}$ & 1 week & 2 weeks & 3 weeks & 4 weeks \\
\hline Very well & 0 & 0 & 0 & 3 & 0 & 0 & 0 & 0 \\
\hline Well & 7 & 3 & 1 & 11 & 8 & 6 & 9 & 8 \\
\hline Moderate & 67 & 75 & 67 & 61 & 73 & 70 & 61 & 73 \\
\hline $\mathrm{Bad}$ & 10 & 6 & 8 & 7 & 3 & 7 & 12 & 3 \\
\hline Very bad & 0 & 0 & 8 & 2 & 0 & 1 & 2 & 0 \\
\hline
\end{tabular}

The frequency of "skin condition" was not changed significantly in week 2,3 , and 4 compared to week 1.

\section{Effects on Oxidative Stress Marker}

Table 5 shows the results of measurement of a free radical oxidative injury index, d-ROMs. The dROMs value significantly decreased after DBB ingestion compared to before ingestion $(p<0.05)$, but no significant change was noted in the placebo group.

\section{Effects on Subjective Symptoms}

Changes in the replies were analyzed in weeks 1 to 4 , and no significant changes were noted in the frequency of feeling fatigue, anxiety and stress, and skin condition in either test diet group (Tables 6-8).

In the replies related to "visual fatigue" and "shoulder stiffness," changes were noted only in the DBB group (Tables 9 and 10). No change was noted in the frequencies of replies related to visual fatigue in the placebo group. In the DBB group, the frequency of "felt visual fatigue frequently" tended to decrease in week 4 of DBB ingestion compared to week $1(p<0.1$; Table 9). Similarly, no significant time-course changes were noted in the frequency of the reply related to shoulder stiffness in the placebo group, while the frequency of "felt shoulder stiffness very frequently" significantly decreased in week 3 compared to week 1 in the DBB group ( $p<0.05$; Table 10).

\section{Correlation Analysis}

To investigate the relationship between improvement in blood fluidity and decreased oxidative stress, we analyzed the correlations between the 
Table 9. Changes in Subjective Symptoms after Placebo or DBB Ingestion (Number of Replies Related to Visual Fatigue)

\begin{tabular}{|c|c|c|c|c|c|c|c|c|}
\hline & \multicolumn{4}{|c|}{ Placebo } & \multicolumn{4}{|c|}{$\mathrm{DBB}$} \\
\hline & 1 week & 2 weeks & 3 weeks & 4 weeks & 1 week & 2 weeks & 3 weeks & 4 weeks $^{a)}$ \\
\hline Very frequently & 8 & 7 & 8 & 8 & 0 & 0 & 3 & 0 \\
\hline Frequently & 21 & 16 & 24 & 8 & 34 & 23 & 15 & 16 \\
\hline Moderate & 35 & 48 & 44 & 49 & 34 & 43 & 51 & 54 \\
\hline Rarely & 20 & 13 & 7 & 16 & 14 & 17 & 15 & 14 \\
\hline Not at all & 0 & 0 & 1 & 3 & 2 & 1 & 0 & 0 \\
\hline
\end{tabular}

a) The frequency of "felt visual fatigue" tended to decrease in week 4 compared to week $1(p<0.1)$.

Table 10. Changes in Subjective Symptoms after Placebo or DBB Ingestion (Number of Replies Related to Shoulder Stiffness)

\begin{tabular}{|c|c|c|c|c|c|c|c|c|}
\hline & \multicolumn{4}{|c|}{ Placebo } & \multicolumn{4}{|c|}{ DBB } \\
\hline & 1 week & 2 weeks & 3 weeks & 4 weeks & 1 week & 2 weeks & 3 weeks $^{a)}$ & 4 weeks \\
\hline Very frequently & 11 & 10 & 12 & 9 & 13 & 10 & 2 & 4 \\
\hline Frequently & 15 & 15 & 22 & 11 & 24 & 23 & 22 & 28 \\
\hline Moderate & 46 & 52 & 39 & 47 & 40 & 47 & 54 & 40 \\
\hline Rarely & 12 & 7 & 10 & 14 & 7 & 4 & 6 & 12 \\
\hline Not at all & 0 & 0 & 1 & 3 & 0 & 0 & 0 & 0 \\
\hline
\end{tabular}

a) The frequency of "felt severe shoulder stiffness" significantly decreased in week 3 compared to week $1(p<0.05)$.

changes in blood fluidity and changes in d-ROM score during DBB and placebo ingestion. Among subjects with a d-ROMs score $>320$, regarded as being in a state of oxidative stress, changes in blood fluidity tended to correlate with changes in d-ROMs score $(\rho=0.55, p=0.06, n=13)$, showing that blood fluidity may have improved in subjects whose oxidative stress was markedly decreased.

\section{DISCUSSION}

Peripheral blood circulation plays an important role in maintaining organ function by facilitating the exchange of oxygen and nutrients between blood and tissues. The diameter of capillary vessels varies among tissues, with a mean of $6 \mu \mathrm{m}$, while the diameters of red and white blood cells are $8 \mu \mathrm{m}$ and $10-25 \mu \mathrm{m}$, respectively, indicating that blood cells have to deform to pass through capillary blood vessels. ${ }^{1)}$ Erythrocytes form the largest group of cells in the blood, and they are highly deformable. The membrane plays an important role in maintaining the flexibility for normal erythrocyte deformability, however, oxidative damage to the erythrocyte membrane has been suggested to induce impairment in these cells' physiological functions, including deformability. ${ }^{20,21,29,30)}$ Diet and general health status are closely related to the morphological and rhe- ological characteristics of erythrocytes. ${ }^{19,28)}$ In this study, daily DBB ingestion significantly decreased the passage time of the specified volume of blood through the microchannels that simulate the human blood capillaries, suggesting that DBB ingestion improved blood fluidity (Table 5). Therefore, DBB intake may improve microcirculation. It was found that single-dose DBB ingestion had an acute dose-dependent effect on peripheral blood flow in healthy subjects. ${ }^{31)}$ Although there is a difference between the ingestion period, single ingestion or 4weeks daily ingestion, DBB single ingestion might increase peripheral blood flow due to its effect of improving peripheral blood fluidity.

Erythrocytes oxidized via reactive oxygen species would be more prone to form aggregates and increase the viscosity of the bloodstream, and reactive oxygen species might impair blood flow in the microcirculation. ${ }^{31)}$ The d-ROMs score, known as a biomarker of oxidative stress, was measured before and after the test diet ingestion. The dROMs score significantly decreased during DBB ingestion (Table 5). It decreased in the placebo group, however, its change was not statistically significant and the reason why placebo ingestion decrease the value is uncertain. Therefore, we should draw conclusions from this result with caution. In our previous studies, DBB was shown to decrease the urinary amount of $8-\mathrm{OHdG}$ which is known as 
a biomarker of oxidative DNA injury. ${ }^{32,33)}$ Fourweek DBB ingestion decrease the urinary $8-\mathrm{OHdG}$ contents from $12.1 \pm 5.1 \mathrm{ng} / \mathrm{mg}$ creatinine (CRE) to $9.6 \pm 4.5 \mathrm{ng} / \mathrm{mg}$ CRE (mean \pm S.D., $n=27, p<$ $0.001 v s$. before ingestion), while there was not significant change between before and after ingestion of water $(12.6 \pm 6.6$ to $12.4 \pm 8.1 \mathrm{ng} / \mathrm{mg} \mathrm{CRE}$, not significant $v s$. before ingestion) in elderly subjects. ${ }^{34)}$ In addition to that study, we found that twoweek DBB ingestion decrease the amounts of 8$\mathrm{OHdG}$ which were excreted for $24 \mathrm{hr}$ in urine from $16.6 \pm 0.6 \mathrm{mg}$ to $14.5 \pm 0.8 \mathrm{mg}$ (mean $\pm \mathrm{SEM}, n=$ $29, p=0.029 v s$. before ingestion), while placebo ingestion did not decrease the level $(15.0 \pm 0.7 \mathrm{mg}$ to $16.1 \pm 1.2 \mathrm{mg}$, mean $\pm \mathrm{SEM}, n=29$, not significant $v s$. before ingestion) in healthy female. ${ }^{35)}$ Since DBB contains abundant histidine and anserine, which are characteristic to DBB (Table 2), ${ }^{36)}$ and these compounds are also reported to have antioxidative actions in vitro, ${ }^{37)}$ it is possible that the observed effect of DBB is derived from the action of these substances. Taken together with thses results, it is highly likely that DBB exhibits an antioxidative effect in vivo. To investigate the relationship between the improvement of blood fluidity and the decrease of oxidative stress, we analyzed the correlations between the changes in the passage with a dROMs score > 320 Carr. U., regarded as indicating a state of oxidative stress. ${ }^{38)}$ Changes in the passage time of $100 \mu \mathrm{l}$ of whole blood tended to correlate with the changes in the d-ROMs score $(\rho=0.55$, $p=0.06, n=13$ ). This result reflected that blood fluidity improved in subjects whose levels of oxidative stress were markedly decreased. There is one possibility that the improvement of blood fluidity was caused by antioxidative activity of DBB.

DBB ingestion was shown to improve mood states and the subjective symptoms of visual fatigue in the previous studies. ${ }^{7-9)}$ DBB was also shown to have an improving effect on the skin by maintaining moisture levels in the skin. ${ }^{10)}$ In the present study, we investigated the effects of DBB on visual fatigue using a questionnaire survey and found that it was observed in only the DBB group (Table 9). This result is consistent with previous observations. ${ }^{7-9)}$ In addition, we newly found that daily DBB intake reduced shoulder stiffness, as a fatigue-related symptom (Table 10). On the other hand, no significant changes were noted in the frequency of feeling fatigue, anxiety and stress, or skin condition in either test diet group (Tables 6-8). The reason why the effect of $\mathrm{DBB}$ on the frequency of feeling fatigue, anxiety and stress, or skin condition was not exhibited in the present study is uncertain, but the method of asking the questions about these effects as well as the subjects were different. In the previous studies, mood states were assessed using the profile of mood states survey, ${ }^{39,40)}$ known as a sensitive measurement of mood states, which were divided into six mood factors (tension-anxiety, depressiondejection, anger-hostility, vigor, fatigue, and confusion), and DBB intake was found to improve the mood states. In the previous study, we tried evaluating the effect of DBB ingestion on the skin condition, we recruited subjects whose skin tended to be dry and rough, while we did not recruit subjects with a view to their skin condition in this study.

Capillary vessels are responsible for oxygen and carbon dioxide gas exchange, nutrient supply, and waste removal. Insufficient blood circulation is considered to lead to the development of shoulder stiffness, ${ }^{2)}$ neck pain, poor circulation, ${ }^{3)}$ and dark skin circles. ${ }^{4)}$ Furthermore, stress has been reported to be closely related to shoulder pain and back pain. ${ }^{41)}$ Taken together with the function of DBB we have already reported and its effect on blood fluidity and oxidative stress marker, we have formulated a hypothesis to explain why DBB is effective against fatigue. It is possible that the antioxidative activity of DBB may improve the oxidative condition of blood vessels or blood cells. Improving blood fluidity may have resulted in a smooth blood supply to the periphery, leading to a more effective functioning of capillary blood vessels. Thus, various symptoms related to fatigue were reduced.

DBB, a hot water extract of bonito muscle, has long been the most familiar soup stock in Japan and is also used as a folk remedy for fatigue. We found that four-week DBB ingestion might exhibit antioxidative activity, ameliorate blood fluidity, and improve some fatigue-related symptoms in healthy subjects. A study to determine what component of DBB affects the blood fluidity and the oxidative stress marker, and through what mechanisms, is now in progress.

Acknowledgement The authors thank Prof. Yasuo Watanabe of Nihon Pharmaceutical University for his helpful discussion, Mr. Masaru Ohkawa of CPCC Co., Ltd., in operating the present study and Ms. Yukiko Satoh of Ajinomoto Co., Inc., for her assistance in data analysis. The authors also appreciate the volunteers who participated in this study. 


\section{REFERENCES}

1) Ganong, W. F. (2006) Review of medical physiology, $22^{\text {nd }}$ ed., Maruzen, Tokyo (in Japanese).

2) Matsumoto, H., Takenami, E., Iwasaki-Kurashige, K., Osada, T., Katsumura, T. and Hamaoka, T. (2005) Effect of blackcurrant anthocyanin intake on peripheral muscle circulation during typing work in humans. Eur. J. Appl. Physiol., 94, 36-45.

3) Takenami, E., Kurashige-Iwasaki, K., Matsumoto, H., Nagasawa, T., Ueda, C., Kitahara, A., Osada, T., Katsumura, T. and Hamaoka, T. (2003) Effects of blackcurrant anthocyanins intake on peripheral circulation during typing workload in humans. J. Jpn. Coll. Angiol., 43, 331-334.

4) Matsumoto, M., Kobayashi, N., Hoshina, O. and Arai, S. (2000) Study on the mechanisms associated with dark circles. J. Soc. Cosmet. Chem. Jpn., 34, 152-159.

5) Kuroda, M., Yamada, K., Nozawa, Y., Ishizaki, T., Hisano, M., Umeki, Y. and Hayabuchi, H. (2005) Anti-fatigue effects of skipjack-tuna extract. Ann. Nutr. Metab., 49, s390.

6) Kuroda, M., Ishizaki, T., Maruyama, T., Takatsuka, Y. and Kuboki, T. (2007) Effect of dried-bonito broth on mental fatigue and mental task performance in subjects with a high fatigue score. Physiol. Behav, in press.

7) Honda, M., Ishizaki, T. and Kuroda, M. (2006) The effect of dried skipjack soup stock on visual fatigue. J. Jpn. Soc. Food Sci. Tech., 53, 443-446.

8) Honda, M., Ishizaki, T., Kajimoto, O., Amano, H., Kajita, M. and Kuroda, M. (2006) Effect of dried skipjack soup stock on asthenopia. Jpn. J. Vis. Sci., 27, 95-101.

9) Honda, M., Ishizaki, T., Kajimoto, O., Amano, H., Kajita, M. and Kuroda, M. (2007) Effect of dried skipjack soup stock on asthenopia in daily life. Jpn. Pharmacol. Ther, 35, 343-348.

10) Yamada, K., Ishizaki, T., Kuroda, M., Kajimoto, O. and Kawagishi, R. (2006) The effect of dried-bonito soup stock intake on drying and roughage of skin. $J$. Nutr. Food, 9, 53-62.

11) Kikuchi, Y., Sato, K., Ohki, H. and Kaneko, T. (1992) Optically accessible microchannels formed in a single-crystal silicon substrate for studies of blood rheology. Microvasc. Res., 44, 226-240.

12) Kikuchi, Y., Sato, K. and Mizuguchi, Y. (1994) Modified cell-flow microchannels in a single-crystal silicon substrate and flow behavior of blood cells. Microvasc. Res., 47, 126-139.

13) Sutton, N., Tracey, M. C., Johnston, I. D., Greenaway, R. S. and Rampling, M. W. (1997) A novel instrument for studying the flow behaviour of erythrocytes through microchannels simulating human blood capillaries. Microvasc. Res., 53, 272281.

14) Chuda, Y., Ono, H., Ohnishi-Kameyama, M., Matsu-moto, K., Nagata, T. and Kikuchi, Y. (1999) Mumefral, citric acid derivative improving blood fluidity from fruit-juice concentrate of Japanese apricot (Prunus nume Sieb. et Zucc). J. Agric. Food. Chem., 47, 828-831.

15) Suganuma, H., Inakuma, T. and Kikuchi, Y. (2002) Amelioratory effect of barley tea drinking on blood fluidity. J. Nutr. Sci. Vitaminol. (Tokyo), 48, 165168.

16) Begum, A. N. and Terao, J. (2002) Protective effect of quercetin against cigarette tar impairment of erythrocyte deformability. J. Nutri. Biochem., 13, 265272.

17) Abdulah, R., Koyama, H., Miyazaki, K., Nara, M. and Murakami, M. (2006) Selenium supplementation and blood rheological improvement in Japanese adults. Biol. Trace Elem. Res., 112, 87-96.

18) Kurihara, T., Deguchi, S., Kato, J., Furukawa, M., Tsuchiya, M., Akimoto, M., Ishiguro, H., Hashimoto, H., Niimi, A., Maeda, A., Shigemoto, M., Yamashita, K., Kawakami, A., Umemura, K., Nakashima, M., Nakano, T. and Saniabadi, A. R. (2001) Impaired blood rheology by remnant-like lipoprotein particles: studies in patients with fatty liver disease. Clin. Hemorheol. Microcirc., 24, 217225.

19) Lee, C. Y. J., Kim, K. C., Park, H. W., Song, J. H. and Lee, C. H. (2004) Rheological properties of erytheocytes from male hypercholesterolemia. $\mathrm{Mi}$ crovasc. Res., 67, 133-138.

20) Davies, K. J. A. and Goldberg, A. L. (1987) Oxygen radicals stimulate intracellular proteolysis and lipid peroxiation by independent mechanisms in erythrocytes. J. Biol. Chem., 262, 8220-8226.

21) Tedesco, I., Russo, M., Russo, P., Iacomino, G., Russo, G. L., Carraturo, A., Faruolo, C., Moio, L. and Palumbo, R. (2000) Antioxidant effect of red wine polyphenols on red blood cells. J. Nutr. Biochem., 11, 114-119.

22) Chung, T. W. and Ho, C. P. (1999) Changes of viscosity of low shear rates and viscoelastic properties of oxidative erythrocyte suspensions. Clin. Hemorheol. Microcirc., 21, 99-103.

23) Cornelli, U., Terranova, R., Luca, S., Cornelli, M. and Alberti, A. (2001) Bioavailability and antioxidant activity of some food supplements in men and women using the D-Roms test as a marker of oxidative stress. J. Nutr., 131, 3208-3211. 
24) Alberti, A., Bolognini, L., Macciantelli, D. and Caratelli, M. (2000) The radical cation of N,Ndiethyl-para-phenylendiamine: a possible indicator of oxidative stress in biological samples. Res. Chem. Intermed., 26, 253-267.

25) Cesarone, M. R., Belcaro, G., Carratelli, M., Cornelli, U., De Sanctis, M. T., Incandela, L., Barsotti, A., Terranova, R. and Nicolaides, A. (1999) A simple test to monitor oxidative stress. Int. Angiol., 2, 127-130.

26) Kamada, H., Hattori, K., Hayashi, T. and Suzuki, K. (2004) In vitro evaluation of blood coagulation activation and microthrombus formation by a microchannel array flow analyzer. Thromb. Res., 114, 195-203.

27) Trotti, R., Carratelli, M. and Barbieri, M. (2002) Performance and clinical application of a new, fast method for the detection of hydroperoxides in serum. Panminerva. Med., 44, 37-40.

28) Kanakaraj, P. and Singh, M. (1989) Influence of hypercholesterolemia on morphological and rheological characteristics of erythrocytes. Atherosclerosis, 76, 209-218.

29) Srour, M. A., Bilto, Y. Y., Jama, M. and Irhimeh, M. R. (2000) Exposure of human erythrocytes to oxygen radicals causes loss of deformability, increased osmotic fragility, lipid-peroxidation and protein degradation. Clin. Hemorheol. Microcirc., 23, 13-21.

30) Hebbel, R. P., Leung, A. and Molandas, N. (1990) Oxidation-induced changes in microrheologic properties of the red blood cell membrane. Blood, 76, 1015-1020.

31) Nozawa, Y., Kuroda, M. and Noguchi, T. (2007) Consumption of dried-bonito broth acutely increases peripheral blood flow in humans. J. Health Sci., 53, 339-343.

32) Shigenage, M., Gimeno, C. and Ames, B. (1989) Urinary 8-hydroxy-2'-deoxyguanosine as a biologi- cal marker of in vivo oxidative DNA damage. Proc. Natl. Acad. Sci. U.S.A., 86, 697-701.

33) Leinonen, J., Lehtimaki, T. and Toyokuni, S. (1997) New biomarker evidence of oxidative DNA damage in patients with noninsulin-dependent diabetes mellitus. FEBS Lett., 417, 150-152.

34) Umeki, Y., Hayabuchi, H., Kuroda, M., Honda, M., Ando, B., Ohta, M. and Ikeda, M. (2006) Effect of the dried-bonito broth (Katsuobushi dashi) on blood pressure and 8-hydroxydeoxyguanosine (8-OHdG), an oxidative stress marker, in the elderly Japanese subjects. J. Hypertention, 24, S379.

35) Nozawa, Y., Ishizaki, T., Kuroda, M. and Noguchi, T. (2007) Effect of dried-bonito broth intake on peripheral blood flow, mood, and oxidative stress marker in humans. Physiol. Behav., in press.

36) Fuke, S. and Konosu, S. (1991) Taste-active components in some foods: a review of Japanese research. Physiol. Behav., 49, 863-868.

37) Kohen, R., Yamamoto, Y., Cundy, K. C. and Ames, B. N. (1988) Antioxidant activity of carnosine, homocarnosine, and anserine present in muscle and brain. Proc. Natl. Acad. Sci. U.S.A., 85, 3175-3179.

38) Cormelli, U., Cormelli, M. and Terranova, R. (1999) Free radicals and vascular disease. The International Union Angiology's Bulletin, 15, 7-10.

39) McNair, D. M. and Lorr, N. (1964) An analysis of mood in neurotics. J. Abnorm. Psychol., 69, 620627.

40) McNair, D. M., Lorr, N. and Droppleman, L. F. (1992) Manual for the profile of mood states (POMS), revised, Educational and Industrial Testing Service (EdITS), San Diego, CA.

41) Takeuchi, T., Nakao, M., Nishitani, M. and Yano, E. (2004) Srress perception and social indicators for low back, shoulder and joint pains in Japan: national surveys in 1995 and 2001. Tohoku J. Exp. Med., 203, 195-204. 